ARTICLE

Received 13 Mar 2015 | Accepted 22 Jun 2015 | Published 24 Jul $2015 \quad$ DOl: 10.1038/ncomms8875 OPEN

\title{
Intrinsically disordered proteins drive membrane curvature
}

David J. Busch', Justin R. Houser', Carl C. Hayden ${ }^{1,2}$, Michael B. Sherman ${ }^{3}$, Eileen M. Lafer ${ }^{4}$

\& Jeanne C. Stachowiak ${ }^{1,5}$

Assembly of highly curved membrane structures is essential to cellular physiology. The prevailing view has been that proteins with curvature-promoting structural motifs, such as wedge-like amphipathic helices and crescent-shaped BAR domains, are required for bending membranes. Here we report that intrinsically disordered domains of the endocytic adaptor proteins, Epsin1 and AP180 are highly potent drivers of membrane curvature. This result is unexpected since intrinsically disordered domains lack a well-defined three-dimensional structure. However, in vitro measurements of membrane curvature and protein diffusivity demonstrate that the large hydrodynamic radii of these domains generate steric pressure that drives membrane bending. When disordered adaptor domains are expressed as transmembrane cargo in mammalian cells, they are excluded from clathrin-coated pits. We propose that a balance of steric pressure on the two surfaces of the membrane drives this exclusion. These results provide quantitative evidence for the influence of steric pressure on the content and assembly of curved cellular membrane structures.

\footnotetext{
${ }^{1}$ Department of Biomedical Engineering, The University of Texas at Austin, 107 W Dean Keeton, Austin, Texas 78712, USA. ${ }^{2}$ Sandia National Laboratories, 7011 East Avenue, Livermore, California 94550, USA. ${ }^{3}$ Department of Biochemistry and Molecular Biology, University of Texas Medical Branch, 1.224 Medical Research Building, Galveston, Texas 77555, USA. ${ }^{4}$ Department of Biochemistry and Center for Biomedical Neuroscience, The University of Texas Health Science Center at San Antonio, UTHSCSA Biochemistry 415B, 7703 Floyd Curl Drive, San Antonio, Texas 78229, USA. ${ }^{5}$ Institute for Cellular and Molecular Biology, The University of Texas at Austin, 107 W Dean, Keeton, Texas 78712, USA. Correspondence and requests for materials should be addressed to J.C.S. (email: jcstach@austin.utexas.edu).
} 
O wing to their critical role in membrane traffic and their accessibility on the plasma membrane surface ${ }^{1,2}$, clathrin-coated pits are a model system for studying cellular membrane curvature. Within these highly curved membrane invaginations, transmembrane cargo molecules are linked to the growing clathrin coat by accessory and adaptor proteins, which bind simultaneously to clathrin, cargo and membrane lipids ${ }^{3}$. It is broadly accepted that the clathrin lattice, which assembles spontaneously into highly curved cages $^{4-6}$, and is important for cell growth and viability ${ }^{7}$, plays a key role in driving the curvature of coated pits. However, several studies have demonstrated that the stably folded membraneinteracting domains of adaptors contain structural motifs that can promote membrane curvature, including wedge-like amphipathic helices ${ }^{8}$ and crescent-shaped BAR domains ${ }^{9}$. In contrast, the protein domains that interact with clathrin and other components of the coat, typically the C-terminal domains (CTDs), are often intrinsically disordered ${ }^{10-12}$, including the CTDs of Epsin1, AP180 and CALM (432, 569 and 339 disordered amino acids in rat sequences, respectively), as well as in the clathrin-uncoating protein auxilin $(267$ disordered amino acids $)^{13}$. Overall, intrinsic disorder is remarkably high in the clathrin endocytic pathway ${ }^{14}$, where it was recently reported that $30 \%$ of proteins contain disordered regions of $>100$ amino acids, including the adaptors above as well as other highly studied proteins such as dynamin, amphiphysin and EPS15 (ref. 15).

Since disordered adaptor domains lack three-dimensional structure and do not interact directly with membranes, they have not been considered as potential drivers of membrane curvature. However, we have recently demonstrated that the pressure generated by collisions among highly crowded proteins of arbitrary structure is sufficient to drive bending ${ }^{16,17}$. Specifically, collisions among crowded molecules produce steric pressure ${ }^{18,19}$ that favours expansion of the membrane surface ${ }^{20,21}$. If this pressure is not balanced by equally crowded molecules on the opposite membrane surface, the resulting imbalance will drive membrane bending towards the more crowded surface. This hypothesis, which has been cited to explain membrane bending during coated vesicle assembly ${ }^{22-25}$, amyloid formation $^{26}$ and autophagy ${ }^{27}$, suggests that proteins with large hydrodynamic radii should make the largest contribution to membrane crowding, since they occupy the most area on the membrane surface ${ }^{20}$. From this perspective, intrinsically disordered protein domains, which have significantly larger hydrodynamic radii than structured proteins of similar molecular weight ${ }^{28}$, could serve as potent drivers of membrane curvature through crowding. For example, the folded Epsin 1 ENTH domain occupies an area of $\sim 16 \mathrm{~nm}^{2}$ on the membrane surface $^{29}$, while the disordered CTDs of Epsin1 and AP180 are expected to occupy 70 and $90 \mathrm{~nm}^{2}$, respectively, based on analytical modelling 28 and experiments ${ }^{11}$. Nonetheless, since the established view has been that structured domains are responsible for curvature generation ${ }^{1}$, the field has overlooked the potential of intrinsically disordered domains to drive membrane curvature.

This work uses a combination of in vitro biophysical studies and quantitative experiments in live cells to investigate the ability of intrinsically disordered domains to create steric pressure on membrane surfaces. We find that disordered adaptor domains drive membranes to form highly curved tubules with considerably greater efficiency than structured domains such as the ENTH domain. Further, by investigating the diffusivity of membranebound adaptor proteins, we demonstrate that the large hydrodynamic radii of intrinsically disordered domains significantly enhances molecular crowding, explaining their ability to drive bending. To test the impact of molecular crowding on the assembly of coated vesicles, we examine the ability of clathrincoated pits to internalize cargos that display intrinsically disordered domains on the extracellular plasma membrane surface. These experiments provide quantitative evidence that large, crowded cargo molecules exert substantial steric pressure on clathrin-coated pits, markedly decreasing the quantity of cargo molecules that can be encapsulated, in comparison with a small, globular control cargo.

\section{Results}

Intrinsically disordered proteins drive membrane curvature. To determine whether intrinsically disordered domains could produce highly curved membrane structures, we incubated histagged proteins, including Epsin1 full-length (FL), Epsin1 CTD and AP180 CTD with $200 \mathrm{~nm}$ diameter small unilamellar vesicles (SUVs) (Fig. 1b) containing DOGS-Ni-NTA (1,2-dioleoyl-snglycero-3-[( $N$-(5-amino-1-carboxypentyl)iminodiacetic acid)succinyl] (nickel salt)) lipids, which bind with high affinity to histidine residues. We examined the resulting structures using transmission electron microscopy. As a positive control for membrane tubulation, we also examined vesicles incubated with the ENTH domain, which has been described as the curvatureinducing domain of Epsin1 (ref. 8). Our recent results demonstrated that the ENTH domain has an equal capacity to drive membrane curvature whether the wild-type protein binds to $\mathrm{PI}(4,5) \mathrm{P}_{2}$-containing membranes using its amphipathic $\mathrm{N}$ terminal helix, (helix0), or an engineered histidine tag that replaces the helix binds DOGS-Ni-NTA containing membranes ${ }^{17}$. Specifically, the his-tagged mutant without helix0, abbreviated here as ENTH $\Delta \mathrm{HO}$, created lipid tubules with the same range of diameters and required the same number of membrane-bound protein molecules per membrane area to drive tubulation as the wild-type ENTH domain. As expected, incubating SUVs with ENTH $\triangle \mathrm{HO}$ produced highly curved membrane tubules (Fig. 1c) ${ }^{17}$. Similarly, Epsin1 FL, Epsin1 CTD and AP180 CTD were all able to generate highly curved membrane tubules when exposed to SUVs containing the same concentration of DOGS-Ni-NTA lipids, $20 \mathrm{~mol} \%$ (Fig. 1d-g; Supplementary Fig. 1).

To measure the efficiency of membrane bending by disordered adaptor domains and compare it with the efficiency of bending by the structured ENTH domain, we employed a giant unilamellar vesicle (GUV) tubulation assay. This assay enables rapid screening of large populations of vesicles and dynamic visualization of lipid tubules using fluorescence microscopy. We varied the concentration of DOGS-Ni-NTA lipids to vary the concentration of his-tagged proteins bound to the membrane surface. Consistent with our previous results, ENTH $\Delta \mathrm{HO}$ was able to tubulate GUV membranes (Fig. 1h-i; Supplementary Fig. 2a) ${ }^{17}$. For all proteins, the fraction of GUVs forming tubules on protein addition increased with increasing DOGS-Ni-NTA concentration (Fig. 1h), even driving some vesicles to collapse at high concentrations (Supplementary Movie 2). However, while $20 \mathrm{~mol} \%$ DOGS-Ni-NTA was required for strong tubulation by the ENTH domain, Epsin1 FL, Epsin1 CTD and AP180 CTD were all able to strongly tubulate GUVs containing just $5 \mathrm{~mol} \%$ DOGS-Ni-NTA (Fig. 1h-i; Supplementary Fig. 2b-g). These results demonstrate that disordered adaptor domains are highly efficient drivers of membrane bending. We next sought to determine the specific mechanism of membrane bending by disordered domains.

IDPs crowd membranes efficiently due to their large radii. Polymer theory ${ }^{28}$ and experimental data ${ }^{11}$ have clearly established that intrinsically disordered proteins have 
a

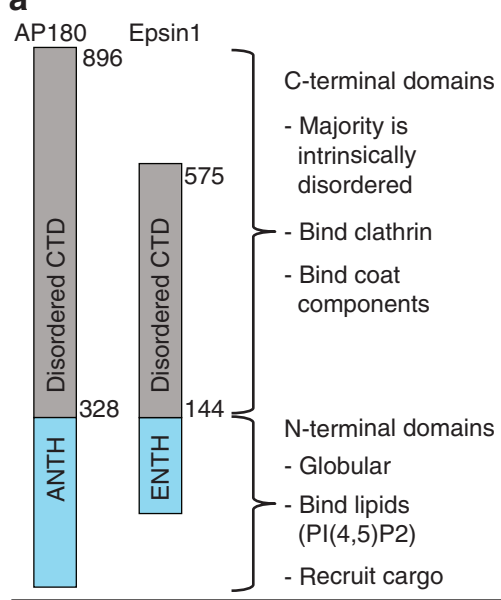

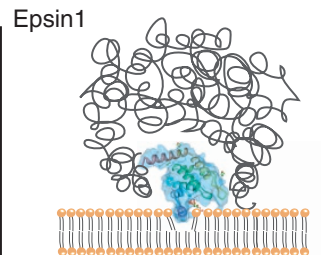

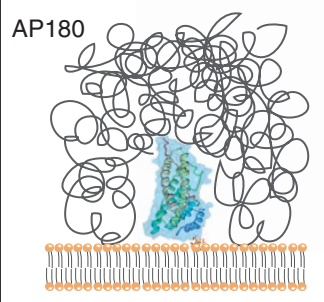

Large IDP domains

drive bending more efficiently

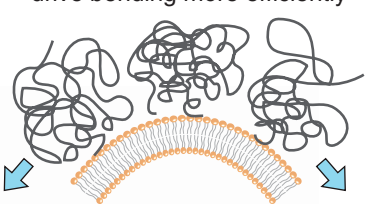

$70-100 \mathrm{~nm}^{2}$ per molecule

Small globular domains drive bending less efficiently

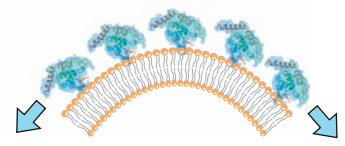

15-20 nm² per molecules
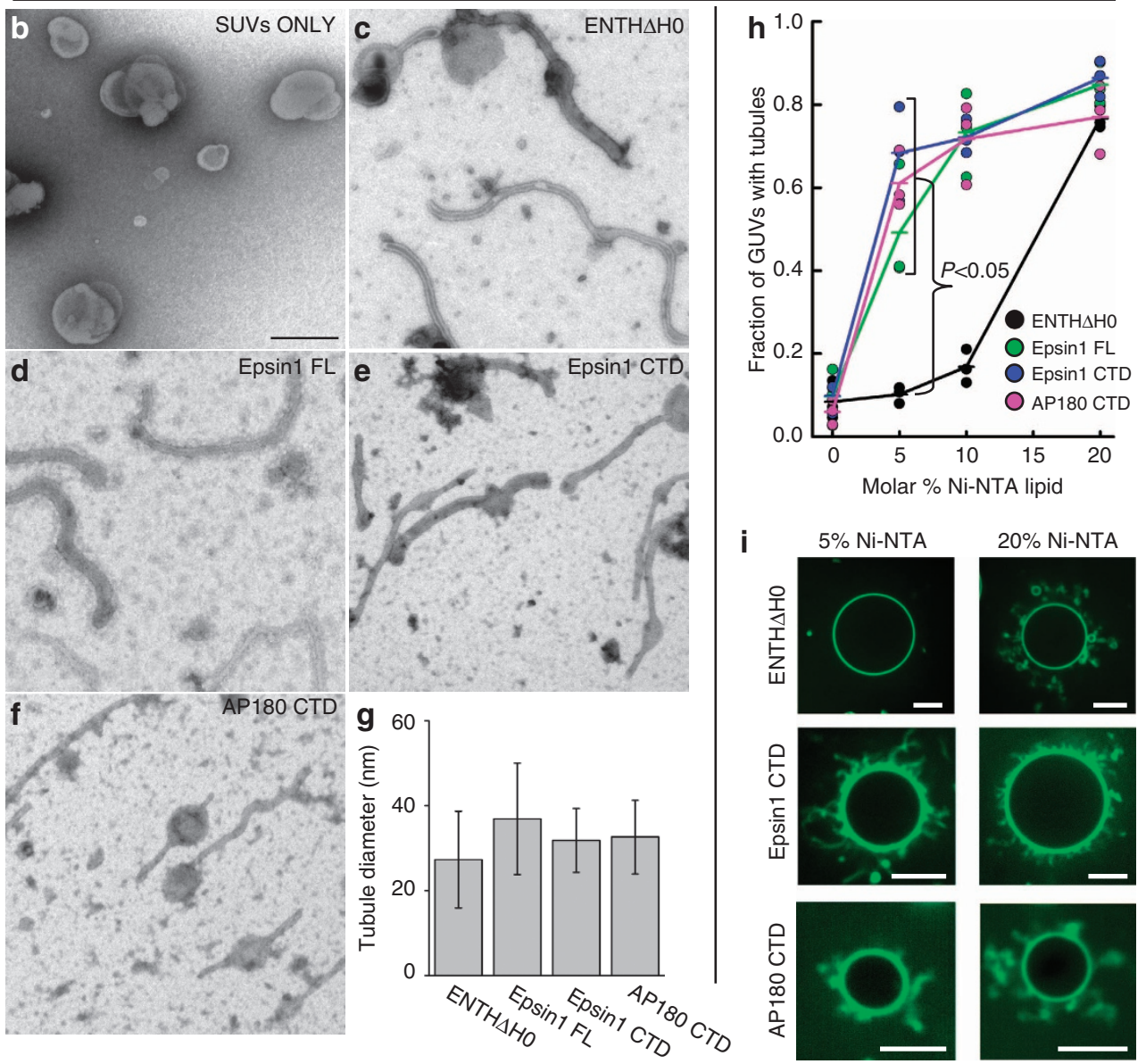

Figure 1 | Disordered adaptor domains drive membrane bending efficiently. (a) The structure of Epsin1 and AP180 consist of stably folded N-terminal domains that bind to membrane surfaces. In contrast the $\mathrm{C}$-terminal domains of these adaptors are intrinsically disordered polypeptides that make multiple bonds with clathrin, other adaptor proteins and various coat components. These domains have large hydrodynamic radii in comparison with $\mathrm{N}$-terminal domains, suggesting they can occupy larger areas on the membrane surface. (b-f) Transmission electron micrographs of initially spherical DOPC vesicles (SUVs) containing 20 mol\% DOGS-Ni-NTA (b) mixed with the following histidine-tagged protein constructs: (c) ENTH $\Delta H O$, (d) Epsin1 FL, (e) Epsin1 CTD and (f) AP180 CTD. Scale bar is $200 \mathrm{~nm}$ and applies to all EM images. (g) All constructs drove membrane tubulation, with average tubule diameters in the range of $15-50 \mathrm{~nm}$. Experimental data is from two electron microscope grids per protein. Error bars represent the mean $\pm \mathrm{s}$.d.; ENTH $\Delta \mathrm{HO}, n=108$ tubules; Epsin1 FL, $n=255$ tubules; Epsin1 CTD, $n=121$ tubules; and AP180 CTD, $n=81$ tubules. (h) Fraction of POPC/DOGS-Ni-NTA GUVs that form tubules following binding of histidine-tagged proteins labelled with Atto488 fluorescent dye. Proteins were added at a concentration of $5 \mu \mathrm{M}$. Proteins that contained disordered domains (Epsin1 FL, Epsin1 CTD and AP180 CTD) each drove the majority of GUVs to form tubules when 5 mol\% DOGS-Ni-NTA was present, while the ENTH $\Delta \mathrm{HO}$ domain required 20 mol\% DOGS-Ni-NTA lipids. $n=3$ independent experiments, $>100$ GUVs per experiment. ENTH $\Delta H 0$ tubulates membranes significantly less than each of the other constructs at $5 \mathrm{~mol} \%$ DOGS-Ni-NTA (Student's $t$-test, unpaired two-tail, $P<0.05$ ). (i) Representative spinning disc confocal fluorescence images of GUVs containing 5 and 20 mol\% DOGS-Ni-NTA after incubation with ENTH $\Delta \mathrm{HO}$, Epsin1 CTD and AP180 CTD, respectively. Scale bar, $10 \mu \mathrm{m}$. 
significantly larger hydrodynamic radii than globular proteins of equivalent molecular weight, suggesting that disordered adaptors will crowd membrane surfaces using fewer molecules per membrane area than structured domains. Nonetheless, the deformability of disordered domains makes it difficult to predict how much area they can occupy on a highly crowded membrane surface. Therefore, we measured the number of membrane-bound protein molecules required to crowd the membrane surface for the ENTH domain, Epsin1 FL and AP180 CTD. When molecular crowding increases, molecular diffusivity decreases owing to an increased rate of intermolecular collisions that limit molecular motion ${ }^{30,31}$. Accordingly, we would expect that fewer disordered proteins per membrane area are required to drive a decrease in protein diffusivity in comparison with the ENTH domain. To test this hypothesis, we measured protein diffusivity on membrane surfaces as a function of the number of molecules per membrane area. We used fluorescence correlation spectroscopy (FCS) to measure the diffusion time, $\tau$, of histidine-tagged protein molecules bound to the surfaces of planar supported lipid bilayers (SLBs) containing DOGS-Ni-NTA (Fig. 2a; Supplementary Fig. 4a-f; Supplementary Movie 3). To vary the number of molecules per membrane area, $m$, membranes included concentrations of DOGS-Ni-NTA lipids ranging from 1 to $10 \mathrm{~mol} \%$. To calculate the number of molecules per area, we used quantitative, photon counting scans of fluorescence intensity (see Supplementary Methods). For each of our proteins (ENTH $\Delta \mathrm{H} 0$, Epsin1 FL and AP180 CTD), we observed an increase in the density of membrane-bound molecules with increasing DOGS-Ni-NTA concentration (Supplementary Fig. 3g). As membrane surfaces became more crowded, the diffusion of each protein slowed as expected (Fig. 2a,b; Supplementary Fig. 3a-c). For the disordered proteins, the drop in diffusivity occurred more rapidly with increasing protein concentration. For example, at approximately the same concentration of membrane-bound proteins, AP180 CTD diffused $\sim 3.5$ times more slowly than ENTH $\Delta \mathrm{HO}$ (Fig. 2c). Under dilute conditions $(<0.1 \%$ coverage), all of the proteins diffused at similar rates, in the range of $1-1.5 \times 10^{-8} \mathrm{~cm}^{2} \mathrm{~s}^{-1}$ (Fig. 2d; Supplementary Fig. $3 \mathrm{~d}-\mathrm{f}$ ), consistent with earlier observations that protein diffusivity on membrane surfaces is limited by drag forces within the membrane $e^{32,33}$. For all the proteins we studied, the decrease in diffusivity with increasing density of membranebound proteins was approximately linear (Fig. 2d).

These results are in agreement with a simple, first-order description of the impact of molecular crowding on the diffusivity of non-interacting spherical particles. Here diffusivity is predicted a Fluorescence correlation spectroscopy

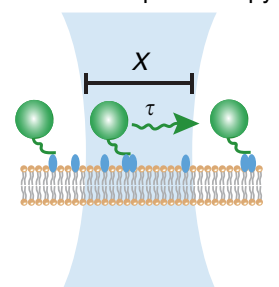

$l=$ DOGS-Ni-NTA

$Q=$ ENTH or IDP

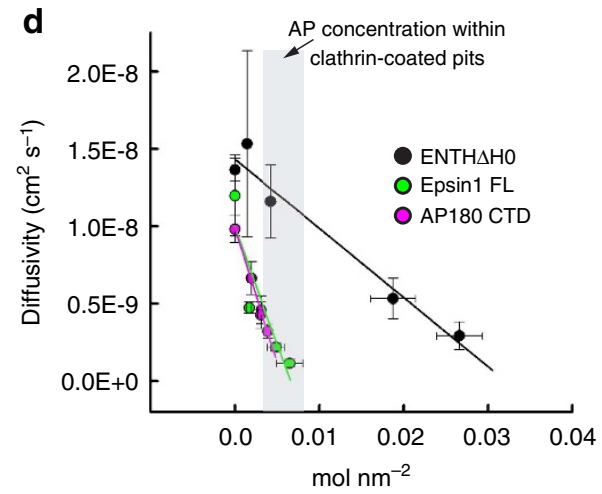

b

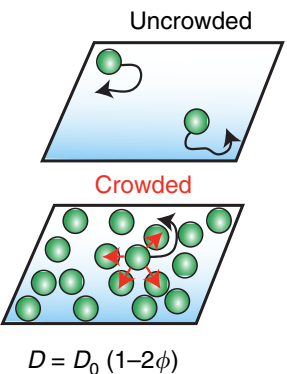

$\phi=\pi R^{2}(m)$

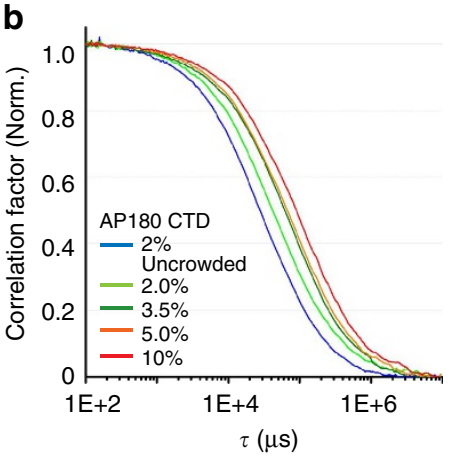

C

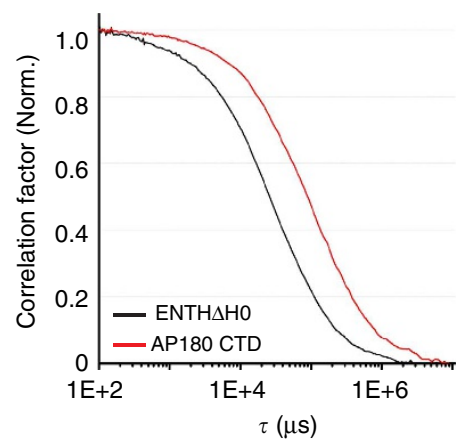

e

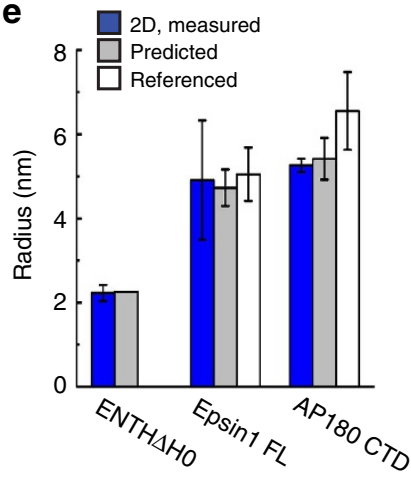

f

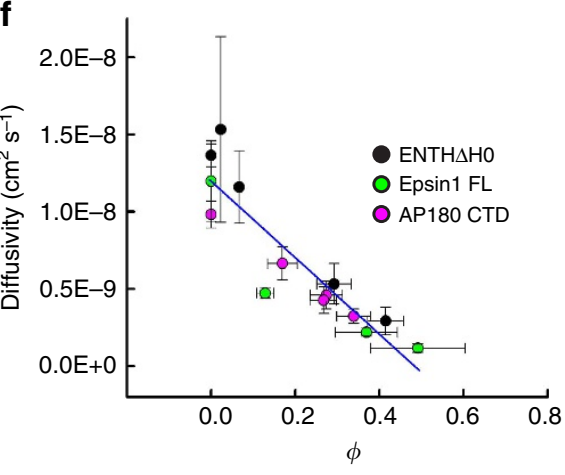

Figure 2 | Intrinsically disordered proteins crowd membrane surfaces efficiently. (a) Fluorescence correlation spectroscopy (FCS) is used to calculate molecular diffusivity by measuring the time $(\tau)$ that a fluorescent molecule (green spheres) takes to pass through a laser focal spot. Supported lipid bilayers (SLB) consisting of POPC lipids with increasing concentrations of DOGS-Ni-NTA lipids (blue ovals) were used to modulate the density of membranebound, histidine-tagged proteins. Diffusivity $(D)$ decreases with molecular coverage, $\phi$ with the relationship $D=D_{\circ}(1-2 \phi)$. $\phi$ can be calculated as the projected area of a protein, $\pi R^{2}$, multiplied by $m$, the number of molecules per $\mathrm{nm}^{2}$. (b) Normalized average FCS curves for AP180 CTD on SLB membranes of increasing molar \% DOGS-Ni-NTA (2-10 mol\%). (c) Normalized average FCS curves for ENTH $\Delta$ HO (black) compared with AP180 CTD (red). AP180 CTD has a slower time of diffusion despite similar number of molecules per area, $\sim 4 \times 10^{-3}$ molecules per nm ${ }^{2}$. Experimental $n$ values for all FCS data is described in methods. (d) Protein diffusivity as a function of the concentration of membrane-bound proteins. Error bars represent the mean \pm s.d. Experimental $n$ values for all membrane coverage data is described in Supplementary Methods. (e) Calculated hydrodynamic radii, $R$, from 2D FCS and photon scanning measurements (b-d), predicted values (ENTH crystal structure or IDP polymer estimates) and referenced gel filtration/analytical centrifugation results ${ }^{11}$. Error bars are the mean \pm s.d. for 2D measurements, and for referenced measurements. Error bars for predicted sizes represent the range of persistence length values. (f) Diffusivity as a function of molecular occupancy, $\phi$. All proteins collapse onto a single linear curve. Error bars represent the mean \pm s.d. 
to decrease linearly with increasing fractional molecular occupancy, $\phi$, according to the relationship, $D=D_{0}(1-2 \phi)$, where $D_{0}$ is the free diffusivity of non-crowded particles (Fig. 2a) ${ }^{34}$. On a membrane surface, $\phi$ is equivalent to the product of the area that an individual protein covers on the membrane surface and the number of molecules per membrane area, $m$. Approximating the molecular area as $\pi R^{2}$, where $R$ is the protein radius, the relationship between diffusivity and the number of molecules per membrane area can be written, $D=D_{0}\left(1-2 \pi R^{2} m\right)$. Fitting this relationship to our data yields estimates of the molecular radii, which are in good agreement with the hydrodynamic radii of Epsin 1 CTD and AP180 CTD (Fig. 2e) based on theoretical predictions (see Methods), measurements in the literature ${ }^{11}$ and our own dilute solution FCS measurements (Supplementary Fig. 3h). Further, plotting diffusivity as a function of fractional molecular occupancy, $\phi$, causes data from all proteins to collapse onto a single linear trend with a negative slope of magnitude approximately twice the average uncrowded diffusivity, as predicted by the theory (Fig. 2f). These results demonstrate that the simple theory of crowding among non-interacting spherical particles can reasonably predict the behaviour of crowded proteins on membrane surfaces. Notably, for the highest values of fractional molecular occupancy, $\phi$, the correlation with diffusivity begins to diverge from the linear trend, likely owing to more complex processes such as molecular aggregation and jamming (Supplementary Fig. 3d,e).

The combined density of all adaptor proteins (AP-2, AP180, Epsin 1 and others) within clathrin-coated pits has been estimated to be $\sim 2-3$ per clathrin triskelia ${ }^{35,36}$, which is $\sim 1$ adaptor per $60-90 \mathrm{~nm}^{2}$ of the membrane surface, based on the physical parameters of clathrin-coated vesicles ${ }^{37}$. Significantly, at this molecular density, ENTH $\Delta \mathrm{HO}$ displays nearly uncrowded diffusion, while AP180 CTD displays highly crowded diffusion (grey box Fig. 2d). These data suggest that disordered adaptor domains generate substantial steric pressure at concentrations found within clathrin-coated pits (Figs 1a and 2d).

Crowded IDPs apply pressure within clathrin-coated pits. Having demonstrated that steric pressure among physiological concentrations of disordered adaptor domains is sufficient to drive membrane bending in vitro, we sought to determine the impact of steric pressure on the structure and content of clathrincoated pits in live cells. Loss of the adaptor AP180 results in an increase in the size of synaptic vesicles, implying decreased membrane curvature, as demonstrated by acute perturbation studies in squid ${ }^{38}$, genetic ablation studies in Drosophila ${ }^{39}$ and Caenorhabditis elegans ${ }^{40}$, as well as RNA interference studies in cultured mammalian hippocampal neurons ${ }^{41}$. These reports suggest that AP180, and other adaptors of similar structure, play a role in shaping coated vesicles. However, the precise physical impact of adaptor CTDs on vesicle curvature is difficult to differentiate from their biochemical role as recruiters of other coat proteins, which may also drive curvature ${ }^{42}$.

Therefore, to isolate the physical impact of disordered adaptor domains on clathrin-coated pits, we have developed a live-cell assay that is based on the concept of molecular competition. Specifically, we reasoned that whether disordered adaptor domains are capable of creating steric pressure that increases membrane curvature when they are present on the coat side (intracellular surface) of the membrane, and then if the same adaptors were present on the cell's external surface as cargo molecules, they ought to generate steric pressure that resists internalization by coated pits.

To test this idea, we developed chimaeric cargo proteins that display disordered adaptor domains on the surfaces of retinal polarized epithelial (RPE) cells. Specifically, chimeras consisted of the short intracellular and transmembrane domains of transferrin receptor (Tf-R), with the extracellular domain completely replaced by an EGFP domain, followed by the CTDs of either Epsin1 or AP180. The resulting cargo molecules are expected to be highly asymmetric, with disordered adaptor CTDs occupying 70-100 $\mathrm{nm}^{2}$ on extracellular plasma membrane surface (Fig. 2e; Fig. 3a,b) and only a few square nanometres on the intracellular surface. For comparison, a control cargo consisted of the same intracellular and transmembrane domains but displayed only an EGFP domain on the plasma membrane surface (Fig. 3a,b). We expect the disordered cargos to have stronger steric interactions with one another and with neighbouring membrane proteins in comparison with the much smaller control cargo. This increase in steric pressure should oppose the internalization of the disordered cargo molecules in clathrincoated pits. Notably, all chimaeric cargo molecules have the same intracellular and transmembrane domains such that they are biochemically identical from the perspective of the cell's clathrin machinery. In contrast to knockout studies, this approach leaves clathrin-mediated endocytosis fully intact, and instead sets up a molecular 'tug of war' in which the physical impact of adaptor CTDs is judged by measuring their ability to resist incorporation into pits.

Further, to evaluate whether the impact of disordered cargos arises from their large hydrodynamic radii, rather than some more specific characteristic of domains derived from endocytic proteins, we created an additional chimaeric transmembrane cargo consisting of the C-terminal intrinsically disordered CTD of neurofilament-M, a protein with similar size to Epsin1 CTD (438 versus 432 amino acids), yet no role in membrane bending. Neurofilaments are polymeric structural filaments that run the length of neuronal axons. Like bottlebrushes, neurofilaments consist of a structured N-terminal core with large intrinsically disordered CTDs radiating outward along their length ${ }^{43}$. The intrinsically disordered CTDs of neurofilament proteins have been described as an 'entropic brush,' and are thought to regulate axonal calibre by occupying volume around the filament, creating steric pressure that repels adjacent filaments ${ }^{44}$. On the basis of its large hydrodynamic radius, we would expect a cargo molecule consisting of the neurofilament-M CTD to increase steric pressure within clathrin-coated pits, similar to those consisting of the CTDs of Epsin1 and AP180. Interestingly, neurofilaments within the axon ${ }^{45,46}$ and clathrin coats ${ }^{35,37}$ contain similar numbers of disordered domains per volume, based on experimental measurements of the geometry and molecular stoichiometry of each structure.

When transiently expressed in RPE cells stably expressing mCherry-tagged clathrin light chain (CLC-mCherry), spinning disc confocal fluorescence imaging revealed that all of the chimaeric cargos were trafficked to the plasma membrane surface and localized within clathrin-coated structures (Fig. 3c; Supplementary Fig. 5). Analysing our confocal images, we quantified the amount of each cargo within clathrin-coated structures. This analysis utilized publically available software, which has been demonstrated to precisely identify individual clathrin-coated structures on the plasma membrane surface ${ }^{47}$, by fitting a two-dimensional (2D) Gaussian function to the intensity profile associated with each putative clathrin puncta. This approach identifies the subpixel location and intensity of each diffraction-limited puncta with signal amplitude significantly above the local background.

Using this approach to identify clathrin-coated structures and analyse their relative brightness, there was a wide variation in the amount of CLC fluorescence per structure (Fig. 3d; Supplementary Fig. 6d-f), as would be expected for a 'snapshot' 
a

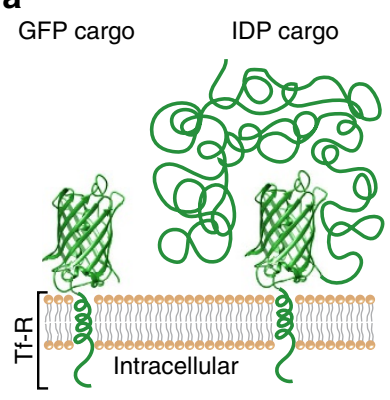

c
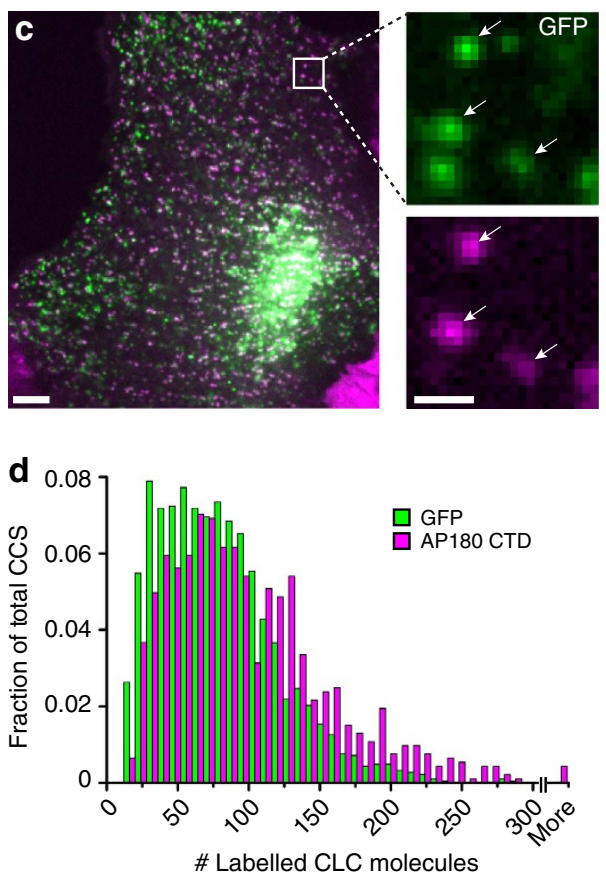

f

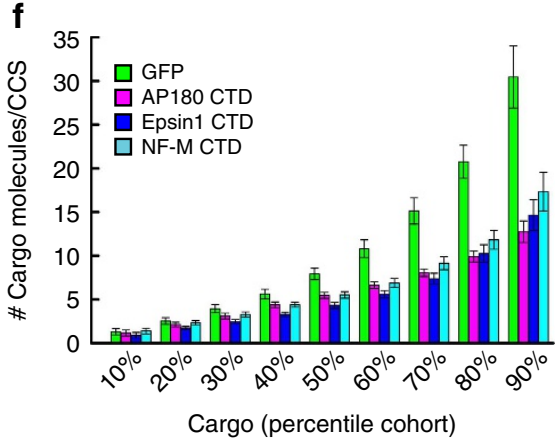

b
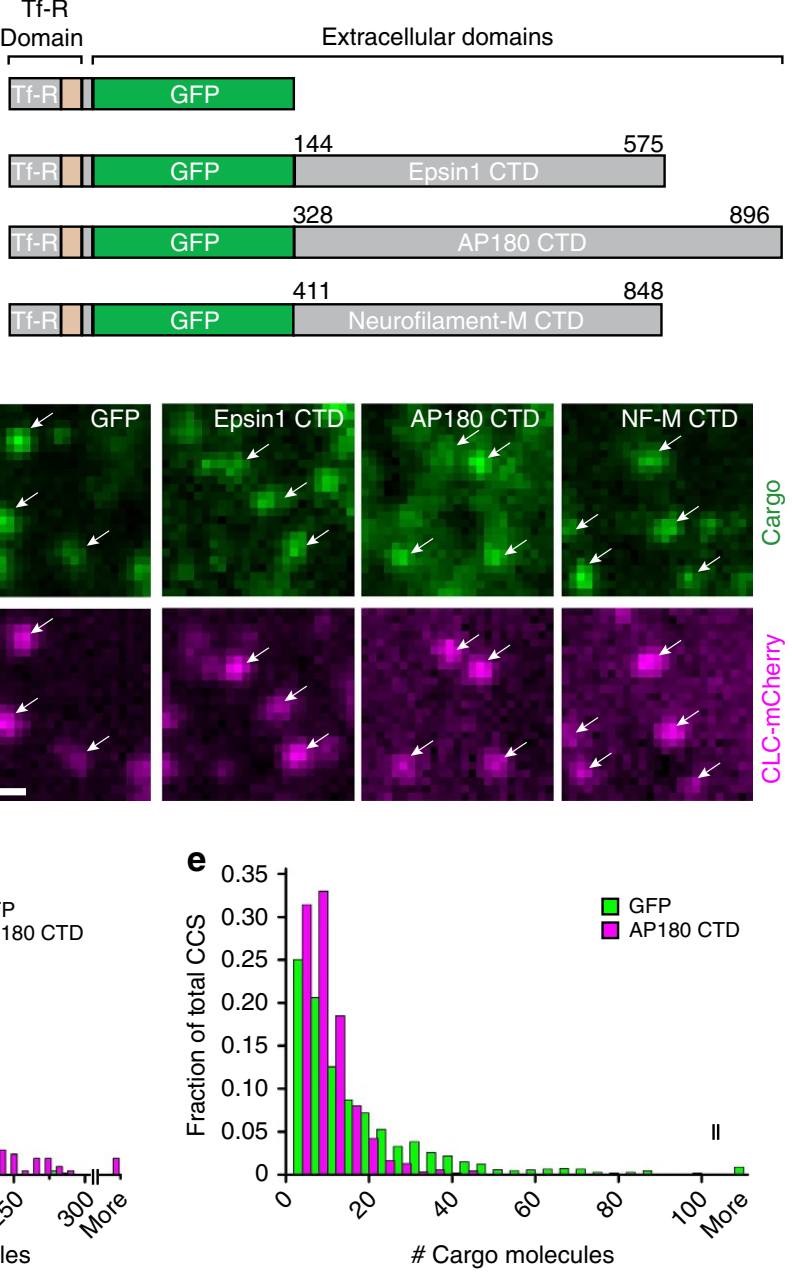

g

Balance of steric forces (Extracellular versus Intracellular)
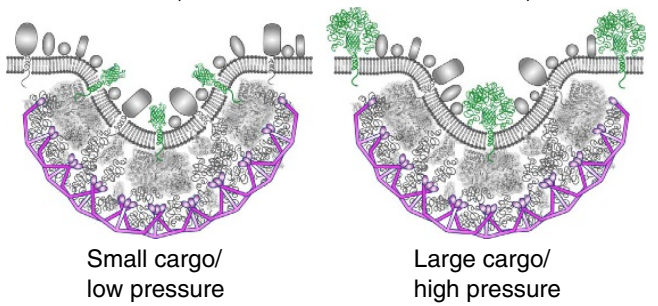

Figure 3 | Crowded adaptor CTDs apply physiologically relevant forces to clathrin-coated structures. (a) Chimaeric IDP cargo used in cell expression experiments. The control GFP cargo (left) consists of the intracellular and transmembrane domains of the transferrin receptor (amino acids 1-85) containing the YXX $\phi$ internalization motif recognized by the clathrin adaptor AP-2, with an extracellular GFP fluorophore. The IDP proteins Epsin1 CTD, AP180 CTD and neurofilament-M CTD were attached onto the extracellular GFP fluorophore (right) to yield IDP cargo constructs. (b) Domain diagrams and the amino-acid lengths for the GFP and IDP cargo. (c) Disordered protein cargos were transiently overexpressed in retinal pigmented epithelial (RPE) cells stably expressing mCherry-tagged clathrin light chain (CLC). A single-confocal section of an RPE cell (left) expressing GFP cargo, and insets of the individual channels (right). Arrows mark clathrin-coated structures (CCS) (mCherry), and the colocalized cargo (EGFP). Scale bar for the whole-cell image represents $5 \mu \mathrm{m}$. Scale bar for insets represents $1 \mu \mathrm{m}$ and applies to all images. (d) Histogram of number of labelled CLC-mCherry molecules for cells expressing the GFP cargo (green bars, mean \pm s.d. $=74.9 \pm 42.4$ molecules) compared with cells expressing AP180 CTD cargo (magenta bars, mean \pm s.d. $=95.8 \pm 57.9$ molecules). (e) Histogram of the number of cargo molecules per CCS for cells expressing the GFP cargo (green bars, mean \pm s.d. $=16.2 \pm 19.6$ molecules) compared with cells expressing the AP180 CTD cargo (magenta bars, mean \pm s.d. $=7.8 \pm 7.2$ molecules). (f) Number of cargo molecules per CCS, displayed as percentile cohorts for each of the cargo constructs (GFP, green bars; AP180 CTD, magenta bars; Epsin1 CTD, blue bars; and NF-M CTD, cyan bars). Data in $\mathbf{d}-\mathbf{f}$ is from two dishes of cells per condition representing independent transfections. Experimental $n$ values are as follows: GFP, $n=1825$ structures, 17 cells; AP180 CTD, $n=925$ structures, 12 cells; Epsin1 CTD, $n=1047$ structures, 9 cells; and NF-M CTD, $n=1287$ structures, 19 cells. (g) Summary model proposing that intrinsically disordered proteins, when crowded, can exert steric pressure that is sufficient to modulate the content of clathrin-coated pits. 
in time, capturing clathrin structures in all stages of assembly and maturation. Dividing the fluorescence per structure by the single molecule fluorescence of mCherry (see Methods) yielded $\sim 75$ mCherry-labelled CLC molecules per structure for cells expressing the control green fluorescent protein (GFP) cargo. To validate this approach, we used it to analyse SLBs with known concentrations of membrane-bound fluorescent proteins, yielding results in close agreement with independent measurements based on photon counting and FCS (see Methods). Since there are three CLC molecules per triskelia, this measurement suggests there are on average 25 triskelia per structure, which is within the expected range for a growing clathrin-coated $\mathrm{pit}^{37}$. As reported recently, labelled CLC molecules are expected to replace a substantial majority of unlabelled endogenous CLC molecules, owing to their overexpression ${ }^{47}$. However, our results may underestimate coat size owing to incorporation of unlabelled endogenous CLC molecules within each structure. We observed a similar, although slightly higher number of CLC molecules per structure among cells expressing the AP180 and Epsin1 CTD cargos (Supplementary Fig. 6j). This increase could arise from an increase in steric pressure that opposes the formation of the smallest, most highly curved pits, although the trend was not maintained for the Nf-M cargo, and could also arise from changes in pit dynamics that were not examined. In contrast, we observed much more significant differences among cells expressing the cargos when we examined their incorporation into clathrin-coated structures.

Once clathrin-coated structures were identified, a 2D Gaussian was fit to the colocalized fluorescence intensity profile of each EGFP cargo puncta. To control for the overall level of cargo expression within cells, we analysed the mean intensity of plasma membrane fluorescence and selected cells with matched levels of expression (Supplementary Fig. 5e). The number of GFP-labelled cargo molecules per structure was quantified exactly as described above for mCherry-labelled CLC (see Methods). We plotted histograms of the number of EGFP-labelled cargo molecules per structure for the control cargo and the AP180 CTD cargo (Fig. 3e; Supplementary Fig. $6 \mathrm{a}-\mathrm{c}$ ), as well as the ratio of cargo to labelled CLC molecules (Supplementary Fig. 6g-i). All histograms indicate a decaying trend in which clathrin-coated structures incorporating a small number of cargo molecules are more frequent than structures incorporating a large number of cargo molecules. However, when disordered cargo molecules were expressed, the distribution shifted to the left, indicating that significantly fewer cargo molecules were incorporated per clathrin-coated structure. The average and median number of chimaeric cargo molecules per structure were: GFP cargo $(16,9)$, AP180 CTD cargo $(8,6)$, Epsin1 CTD cargo $(8,5)$ and Nf-M CTD cargo $(10,6)$. To highlight the exclusion of disordered cargos from clathrin-coated structures, the data in the histogram is divided into a series of 10 cohorts of equal probability and increasing cargo content. Specifically, all structures were ranked according to the amount of cargo they contained. The bottom $10 \%$ were then averaged and plotted as the $10 \%$ cohort, the next $10 \%$ as the $20 \%$ cohort, and so on. Plotting the data in this way enables comparison of events of equal probability. For example, examining the $10 \%$ cohort, it is equally probable to find a clathrin-coated structure containing just one copy of any of the cargo molecules in our study. In contrast, examining the $90 \%$ cohort reveals that it is equally probable to find a structure that

a AP180 CTD $4380 \quad$ AP180 CTD cargo b truncation cargo Tf-R Domain Extracellular domains

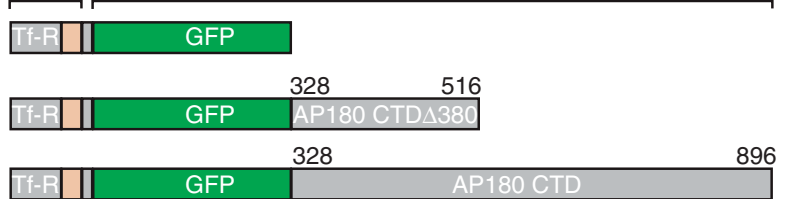
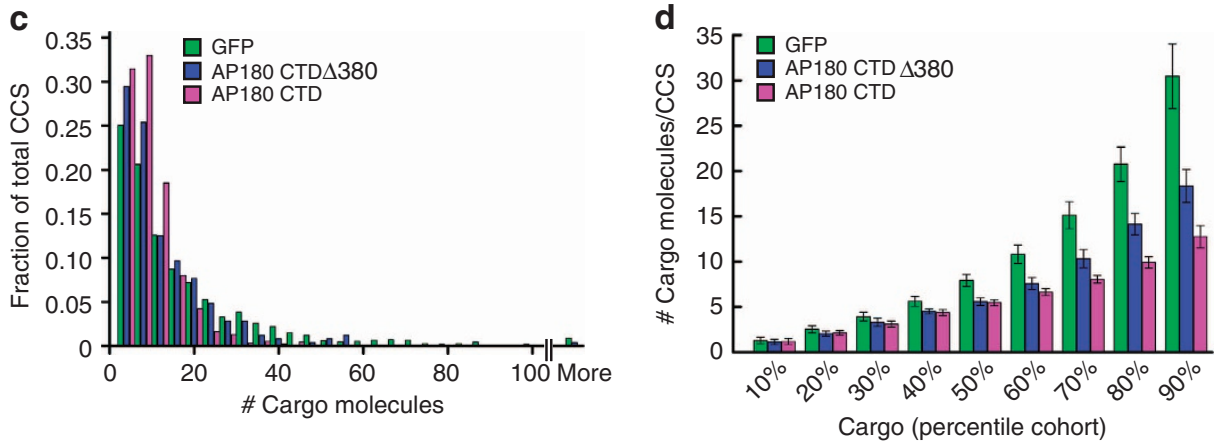

Figure 4 | A truncated AP180 CTD cargo molecule demonstrates size dependent exclusion from clathrin-coated structures. A truncated AP180 CTD cargo molecule was made, which only comprises 188 amino acids of the intrinsically disordered C-terminal domain of AP180. (a) Cartoon schematic demonstrating that the truncated AP180 C-terminal domain (AP180 CTD $\Delta 380$ truncation cargo) should occupy a smaller area on the cell membrane, as compared with the full-length AP180 C-terminal domain (AP180 CTD cargo). (b) Diagram of each chimaeric cargo depicting the amino acid lengths. (c) Number of cargo molecules per clathrin-coated structure (CCS) in RPE cells expressing GFP cargo, AP180 CTD 4380 truncation cargo and AP180 CTD cargo. For GFP and AP180 CTD cargos, the histogram data (c) and percentile cohort data (d) are the same as displayed in Fig. 3f, GFP cargo (green bars, $n=1825$ structures, 17 cells) compared with cells expressing the AP180 CTD cargo (magenta bars, $n=925$ structures, 12 cells). The AP180 CTD $\Delta 380$ truncation cargo is incorporated into clathrin-coated structures to intermediate levels (blue bars, $n=248$ structures, 7 cells), further demonstrating the size dependence of cargo exclusion. Values for each percentile cohort represent the mean number of molecules $\pm \mathrm{s}$. d. 
contains 30 GFP cargo molecules as it is to find one that contains only 12-15 disordered cargo molecules.

These results suggest that clathrin-coated structures can easily incorporate a few large cargo molecules, but cannot incorporate a large number of disordered cargo, likely owing to increased steric pressure on the membrane surface among the large cargo. Notably, the Nf-M CTD cargo follows the same trend, indicating that it arises from a general physical property of disordered protein domains. As an additional confirmation of this size dependence, we developed a truncated AP180 CTD cargo molecule with approximately two-thirds of the CTD removed which we termed AP180 CTD $\Delta 380$ (Fig. 4a,b). This truncated cargo molecule is estimated to occupy a smaller area on the membrane surface. Indeed, this construct was incorporated into clathrin-coated structures to an intermediate degree when compared with the GFP cargo and the FL AP180 CTD cargo molecules (Fig. 4c,d).

\section{Discussion}

Cargo molecules are recruited into clathrin-coated pits by individual adaptor proteins such as AP-2, Epsin1 and AP180. These interactions have relatively low affinities $(\sim 1 \mu \mathrm{M})^{3,48}$, enabling cargo molecules to transiently associate and dissociate with growing pits and to diffuse from adaptor to adaptor within pits ${ }^{49}$. Our data demonstrates that cargo molecules are excluded from clathrin-coated structures as a function of size. This exclusion effect indicates that when large cargos enter pits they create steric pressure that competes with their affinity for cargo adaptors. This reduced binding favours dissociation of large cargo from pits, reducing steric pressure and thereby enabling curved pits that incorporate a reduced number of large cargos to form.

In contrast, adaptor IDP domains within the coat form multiple bonds with other coat components, leading to a nearpermanent affinity for the coated vesicle ${ }^{50}$. Therefore, when adaptor IDP domains become crowded within the coat, the steric pressure they create cannot be relieved by their dissociation. Considering the geometry ${ }^{37}$ and stoichiometry ${ }^{35}$ of an average clathrin-coated vesicle, the number of disordered adaptor and accessory proteins per coated vesicle is around 100, much larger than the typical number of disordered cargo molecules in our studies, $<10$. The observation that a relatively small number of disordered cargo drives steric exclusion suggests that the greater number of disordered domains in the coat creates substantial steric pressure. The precise mechanisms by which this steric pressure can help to drive membrane invagination during the dynamic assembly of a clathrin-coated pit remain to be revealed, providing an exciting topic for future experiments and theoretical modelling.

These results fit into a larger pattern in which disordered polymers frequently generate steric pressure to achieve diverse cellular goals. As discussed above, the disordered C termini of neurofilaments sterically exclude one another to control axonal calibre $^{44}$. Similarly, disordered phenlalanine-glycine repeats of nucleoporin proteins are thought to provide a steric gating mechanism for the nuclear pore ${ }^{51,52}$. Further, the role of disordered polymers in producing steric pressure that prevents nonspecific molecular interactions at biochemical surfaces includes diverse examples from the glycocalyx ${ }^{53}$ to synthetic layers of polyethylene glycol ${ }^{54}$.

While this study has focused on disordered protein domains, other large and highly structured adaptor proteins, such as the tetrameric adaptor protein AP-2 may also generate steric pressure within coats. The structured trunk domains of AP-2 occupy $\sim 80-100 \mathrm{~nm}^{2}$ on the membrane surface ${ }^{55}$, comparable to the disordered adaptor domains. Furthermore, AP-2 is incorporated in pits at approximately a 1:1 stoichiometric ratio with clathrin triskelia ${ }^{35}$, making this protein a major component of pits that could contribute substantially to crowding the membrane surface.

Here we demonstrate that intrinsically disordered domains are a previously unknown class of highly potent membrane bending proteins. By correlating molecular diffusivity with the number of membrane-bound proteins per membrane area, our data demonstrate that the capacity of disordered proteins to bend membranes arises from an enhanced ability to crowd membrane surfaces. Further, live-cell imaging experiments demonstrate that these molecules resist incorporation into clathrin-coated structures when expressed as cargo molecules, owing to steric interactions among them. Consistent with these results, Mettlen et al. have shown that overexpression of a chimaeric LDL receptor cargo drives the formation of clathrin-coated structures of low curvature, including large pits and flat clathrin lattices ${ }^{56}$. Similarly, Liu et al. observed that attachment of large ligands to cargo ectodomains increased the size of clathrin-coated pits ${ }^{57}$. Further, our results may provide a mechanistic explanation for the recent report by Copic et al. that in yeast cells, the COPII coat requires rigid coat components to accommodate large and asymmetric cargo molecules ${ }^{22}$. Finally, our results motivate a re-examination of a recent report by Dannhauser et al. ${ }^{58}$, demonstrating that only clathrin and the disordered CTD of Epsin 1 are required to reconstitute highly curved clathrin-coated pits. These elegant results were interpreted as a confirmation of clathrin's capacity to drive membrane curvature, neglecting the potential role of the disordered domain, an effect that our results demonstrate to be highly significant. Taken together our results support a model in which a dynamic balance of steric pressure between coat and cargo proteins modulates the content and curvature of coated membrane vesicles.

\section{Methods}

Chemical reagents. POPC (1-palmitoyl-2-oleoyl-sn-glycero-3-phosphocholine), DOPC (1,2-dioleoyl-sn-glycero-3-phosphocholine) and DOGS-Ni-NTA (1,2-dioleoyl-sn-glycero-3-[(N-(5-amino-1-carboxypentyl)iminodiacetic acid)-succinyl] (nickel salt))were purchased from Avanti Polar Lipids (Alabaster, AL, USA). Atto488 fluorescent dye NHS ester was purchased from ATTO-TEC. 4-(2hydroxyethyl)-1-piperazineethanesulphonic acid (HEPES), phenylmethanesulfonyl fluoride (PMSF), benzamidine hydrochloride, EDTA-free protease inhibitor tablets and TCEP (Tris(2-carboxyethyl)phosphine hydrochloride) were purchased from Sigma-Aldrich (St Louis, MO, USA). Leupeptin and pepstatin were purchased from Roche (Indianapolis, IN, USA). MOPS (3-( $N$-morpholino)propanesulfonic acid), $\beta$-mercaptoethanol, Triton $\mathrm{X}-100, \mathrm{NaCl}$ and $\mathrm{PBS}$ tablets were purchased from Fisher Scientific.

Plasmid constructs. Plasmid constructs used in this study are as follows: ENTH $\Delta \mathrm{H} 0$ was as previously described ${ }^{17}$. The his-Epsin 1 FL (Epsin1 FL) and his-Epsin1 CTD constructs, (rat Epsin1, amino acids 144-575; NM_057136) as well as the rat his-AP180 CTD construct (rat AP180, amino acids 328-896; CAA48748) were provided as a kind gifts by Dr Ernst Ungewickell. The constructs GST-6His-Epsin1 CTD (Epsin1 CTD) and GST-6his-AP180 CTD (AP180 CTD) were made by subcloning the PCR amplified CTDs from the above templates into the pGex4T2 vector using incorporated restriction sites EcoRI and XhoI yielding a 27 amino-acid linker (including the 6 his tag) between GST and the CTD. A mouse neurofilament-M plasmid was aquired from Addgene (plasmid \#32909;

Cambridge, MA, USA), which includes an intrinsically disordered CTD (amino acids $411-848)^{59}$. The chimaeric Tf-R $\Delta$ Ecto-GFP (GFP cargo) was made by modifying the Tf-R-GFP construct (pEGFP-N1 backbone), kindly provided by Dr Tomas Kirchhausen (Harvard medical school), where the entire Tf-R sequence was excised by digesting with EcoRI and BamHI, and inserting only the PCR amplified intracellular and transmembrane domains of Tf-R adjacent to the GFP fluorophore with EcoRI and BamHI sites. An intermediate construct lacking a stop codon was made by digesting Tf-R $\Delta$ Ecto-GFP with BamHI and NotI to remove GFP, and inserting a mutated PCR amplified GFP with the stop codon (TAA) replaced by a glycine (GGA). For Tf-RAEcto-GFP Epsin1 CTD (Epsin1 CTD cargo), Tf-R $\Delta$ Ecto-GFP AP180 CTD (AP180 CTD cargo) and Tf-R $\Delta$ Ecto-GFP Neurofilament-M CTD (NF-M CTD cargo), the CTDs of each cargo construct were PCR amplifying using primers containing NotI sites. PCR amplified products were then restriction cloned into NotI sites on the intermediate Tf-R vector described above. The AP180 truncation mutant was cloned using site-directed mutagenesis of Tf-R $\Delta$ Ecto-GFP AP180 CTD plasmid with PCR primers that incorporated a premature stop codon at amino-acid position 516 (A516stop) of the 
CTD. Following transformation of the DpnI digested reactions, positive clones were selected. The his-mCherry plasmid was acquired from Addgene (plasmid $\# 29722$ ). All constructs were confirmed by DNA sequencing.

Protein purification. Proteins were purified as previously described ${ }^{17,20}$ with the following modifications: proteins were expressed in BL21(DE3) pLysS cells either overnight at $18^{\circ} \mathrm{C}(\mathrm{ENTH} \Delta \mathrm{H} 0$ and Epsin $1 \mathrm{FL})$, or at $30-37^{\circ} \mathrm{C}$ for $3-6 \mathrm{~h}(\mathrm{Epsin} 1$ CTD and AP180 CTD). Proteins were purified from bacterial extracts either by incubation with DOGS-Ni-NTA agarose in $25 \mathrm{mM}$ HEPES, pH 7.4, $150 \mathrm{mM} \mathrm{NaCl}$, $1 \mathrm{mM}$ PMSF, and $1 \mathrm{mM} \beta-\mathrm{ME}$ or by glutathione agarose in $0.5 \mathrm{M}$ Tris, $0.1 \% \beta-\mathrm{ME}$, $4 \mathrm{mM}$ EDTA, 5\% glycerol, $1 \mathrm{mM}$ PMSF, $1 \mathrm{mM}$ benzamidine hydrochloride, $7.5 \mu \mathrm{M}$ pepstatin and $10 \mu \mathrm{M}$ leupeptin at $\mathrm{pH}$ 8.0. Bacteria cells were lysed with $1 \%$ Triton X-100 and a horn sonicator at $30 \%$ amplitude for $4-6 \mathrm{~min}$ in 30 -s intervals. After extensive washing, proteins were eluted from DOGS-Ni-NTA or glutathione resins by gradually increasing to a final buffer concentration of $200 \mathrm{mM}$ imidazole or $50 \mathrm{mM}$ glutathione, respectively. Eluted proteins were concentrated and dialyzed for completion with either $25 \mathrm{mM}$ HEPES, $\mathrm{pH} 7.4,150 \mathrm{mM} \mathrm{NaCl}$ and $1 \mathrm{mM}$ $\beta$-mercaptoethanol at $4{ }^{\circ} \mathrm{C}$ overnight for DOGS-Ni-NTA agarose purifications, or in $0.5 \mathrm{M}$ Tris, $0.1 \% \beta-\mathrm{ME}$ at $4{ }^{\circ} \mathrm{C}$ overnight for glutathione agarose purifications. Proteins were stored as small aliquots at $-80^{\circ} \mathrm{C}$.

Electron microscopy. SUVs were prepared by drying $20 \mathrm{~mol} \%$ DOGS-Ni-NTA and $80 \mathrm{~mol} \%$ DOPC lipids into a glass test tube. The lipid film was hydrated with $20 \mathrm{mM}$ MOPS, pH 7.35, $200 \mathrm{mM} \mathrm{NaCl}$. Hydrated films were vortexed and extruded through a 200-nm pore filter (Avanti) to create small vesicles of $\sim 200-\mathrm{nm}$ diameter. His-tagged proteins were mixed with $1 \mathrm{mM}$ TCEP and incubated with vesicles for $30 \mathrm{~min}$ at $37^{\circ} \mathrm{C}$ at the indicated protein to DOGS-Ni-NTA lipid molar ratios: ENTH $\Delta$ H0, 1:10; Epsin1 FL, 1:20-40; Epsin1 CTD, 1:20; AP180 CTD, 1:20. In each case, all DOGS-Ni-NTA-binding sites are expected to be bound by protein. Five microlitres of each solution was added to glow discharged 300 mesh carbon-coated formvar grids and stained with $2 \%$ uranyl acetate (Electron Microscopy Sciences; Hatfield, PA, USA). Images were collected from two electron microscope grids per protein condition on a Technai Spirit BioTwin T12 electron microscope (Technai; Hillsboro, OR, USA). Vesicle diameters were measured using ImageJ software.

Giant unilamellar vesicle preparation. GUVs were electroformed according to published protocols ${ }^{17,60}$. Briefly, a lipid composition of POPC with increasing mol\% DOGS-Ni-NTA (5-20 mol\%) was dried as a lipid film, gently hydrated with $350 \mathrm{mOsm}$ sucrose and electroformed at $55^{\circ} \mathrm{C}$ by applying an oscillating potential at $1.4 \mathrm{~V}$ peak to peak for $3 \mathrm{~h}$. Vesicles were diluted sixfold in MOPS buffer $(20 \mathrm{mM}$ MOPS, $\mathrm{pH} 7.35 ; 200 \mathrm{mM} \mathrm{NaCl}$ ) and incubated with his-tagged proteins containing $5 \mathrm{mM}$ TCEP. All buffers were matched in osmolarity to GUVs using a vapour pressure osmometer. Data were collected from three independent batches of GUVs. More than 100 GUVs per condition were imaged, and the number of GUVs with lipid tubules were counted and calculated as a percent of total GUVs. Lipid tubules were defined as highly dynamic, protein-labelled, extended structures of diffraction-limited diameter.

Fluorescence microscopy. Spinning disc confocal microscopy (Zeiss Axio Observer Z1 with Yokagawa CSU-X1M) was used to image GUVs and live cells. The laser wavelengths of 488 and $561 \mathrm{~nm}$ were used for excitation. Emission filters were centred at $525 \mathrm{~nm}$ with a $50-\mathrm{nm}$ width, and $629 \mathrm{~nm}$ with a $62-\mathrm{nm}$ width. A triple pass dichroic mirror was used: $405 / 488 / 561 \mathrm{~nm}$. The microscope objective used was a Plan-Apochromat $100 \times, 1.4$ numerical aperture oil immersion objective. GUVs were imaged on a scientific cMOS PCO edge 4.2 camera (PCO AG; Kelheim, Germany). Live-cell experiments were imaged on a cooled ( $-70 \mathrm{C})$ EMCCD iXon3 897 camera (Andor Technology; Belfast, UK).

Supported lipid bilayer preparation. SUVs were prepared by drying down lipid films in clean glass conical tubes. The tubes were put under vacuum for $2-3 \mathrm{~h}$. Then, the lipid film was resuspended in phosphate buffered saline (PBS) to create a $2 \mathrm{mM}$ solution of liposomes. The liposomes were sonicated with a horn sonicator at $75 \%$ power for $16 \mathrm{~min}$ in $4 \mathrm{~min}$ intervals separated by $2 \mathrm{~min}$ cooling periods in an ice bath. Ultraclean coverslips were used as the substrate for SLB preparation. Silicone gaskets formed a well on the coverslip surface. PBS was used to initially hydrate the coverslip; then a $2 \mathrm{mM}$ solution of SUVs was pipetted onto the coverslip, followed by a $15 \mathrm{~min}$ incubation period during which the SLB formed. The SLB was washed repeatedly with PBS to remove SUVs left in solution. After washing, protein solution was pipetted onto the SLB and incubated for the experiments described below.

Fluorescence correlation spectroscopy. SUVs consisting of POPC lipids and varying percentages of DOGS-Ni-NTA lipids were used to prepare SLBs. A small concentration of Atto488-labelled protein (2-8 $\mathrm{nM}$ depending on the protein and mol\% DOGS-Ni-NTA) was prebound to the SLB surface for 30 min for ENTH $\Delta \mathrm{H} 0$ and Epsin $1 \mathrm{FL}$, and $60 \mathrm{~min}$ for AP180 CTD. The SLB was washed to remove excess fluorescent protein. Then, a $2 \mu \mathrm{M}$ solution of unlabelled protein was added to the
SLB to create a crowded membrane surface (20-min incubation for Epsin1 FL and ENTH $\Delta$ H0, 60-min incubation for AP180). Point FCS measurements were acquired using a custom-built time-correlated single-photon counting confocal microscope that has been described previously ${ }^{20}$. FCS curves were collected for 250-500 s using autocorrelation through Becker and Hickl data acquisition software. Experimental $n$ values for FCS curves are as follows: for ENTH $\Delta \mathrm{H} 0$, $n=9$ curves, 2 SLBs (2\% DOGS-Ni-NTA, uncrowded); $n=14$ curves, 2 SLBs (1\% DOGS-Ni-NTA); $n=12$ curves, 2 SLBs ( $2 \%$ DOGS-Ni-NTA); $n=19$ curves 3 SLBs (5\% DOGS-Ni-NTA); $n=15$ curves, 2 SLBs (7.5\% DOGS-Ni-NTA); and $n=13$ curves, 2 SLBs (10\% DOGS-Ni-NTA); and for Epsin1 FL, $n=10$ curves, 2 SLBs (2\% DOGS-Ni-NTA, uncrowded); $n=6$ curves, 1 SLB (1\% DOGS-NiNTA); $n=11$ curves, 2 SLBs (2\% DOGS-Ni-NTA); $n=9$ curves, 2 SLBs (3.5\% DOGS-Ni-NTA); $n=7$ curves, 2 SLBs (5\% DOGS-Ni-NTA); for AP180 CTD, $n=13$ curves, 2 SLBs (2\% DOGS-Ni-NTA, uncrowded); $n=12$ curves, 2 SLBs ( $2 \%$ DOGS-Ni-NTA); $n=12$ curves, 2 SLBs (3.5\% DOGS-Ni-NTA); $n=17$ curves, 3 SLBs (5\% DOGS-Ni-NTA); and $n=15$ curves, 2 SLBs $(10 \%$ DOGS-Ni-NTA). The autocorrelations obtained were fit with the standard 2D autocorrelation function:

$$
G(t)=\left(1+a e^{-t / \tau_{c}}\right) \times\left(\frac{C}{1+t / \tau_{\mathrm{D}}}\right)+1
$$

where $C$ is $1 / N_{\mathrm{p}}$, and $N_{\mathrm{p}}$ is the average number of diffusing proteins in the excitation region. $\tau_{\mathrm{D}}$ is the diffusion time at which the autocorrelation function reaches one-half its initial amplitude. Corrections for short time processes such as intersystem crossing are included in $a$ and $\tau_{c}$, which are held constant in the fitting They have little effect on fitting the longer time processes of interest here. The autocorrelation curves were fit with a custom nonlinear fitting program using the Levenberg-Marquardt algorithm implemented in ALGLIB (Sergey Bochkanov, www.alglib.net). In crowded Epsin1 FL data sets there was a contribution from labelled protein diffusing in the buffer leading to a fast diffusion component. These data sets were fit using a pseudo three-dimensional (3D) term, which has the form of a $2 \mathrm{D}$ autocorrelation term:

$$
G(t)=\left(1+a e^{-t / \tau_{c}}\right) \times\left(C \times \frac{\text { fraction } 1}{1+t / \tau_{\mathrm{D}_{1}}}\right)+\left(C \times \frac{1-\text { fraction } 1}{1+t / \tau_{\mathrm{D}_{2}}}\right)+1
$$

where fraction 1 is the fraction diffusing with the fixed diffusion time of $\tau_{\mathrm{D} 1}$ for the fast component, $0.8 \mathrm{~ms}$, corresponding to the approximate solution phase diffusion time for this protein. This approximation is appropriate because a $3 \mathrm{D}$ term approaches the form of a 2D term in the limit that the $z$ axis of the focal volume is long. The larger $\tau_{\mathrm{D} 2}$ corresponding to membrane diffusion is reported.

Estimation of hydrodynamic radii based on polymer theory. A first-order estimate of the hydrodynamic radius, $R_{\mathrm{g}}$, of a random, self-avoiding polymer chain can be calculated from the following equation, $R_{\mathrm{g}}=\sqrt{\frac{L_{\mathrm{p}}}{3}}$, in which $l_{\mathrm{p}}$ is the chain persistence length and $L$ is the total length of the fully extended polymer chain. The average persistence length of an intrinsically disordered polypeptide has been measured in the range of $0.4 \pm 0.07 \mathrm{~nm}$ (ref. 28). The total lengths of the disordered domains considered in this study can be approximated by multiplying the length of a single amino acid (that is, the distance between two alpha carbon atoms), $0.38 \mathrm{~nm}$ (ref. 61), by the total number of amino acids in the chain, yielding the following chain length values: AP180 CTD (569 amino acids, $216 \mathrm{~nm}$ ), Epsin1 CTD (432 amino acids, $164 \mathrm{~nm}$ ) and neurofilament-M CTD (438 and $166 \mathrm{~nm})$. Using these values, the equation above yields the following approximate hydrodynamic radii: AP180 CTD $(5.4 \pm 0.5 \mathrm{~nm})$, Epsin1 CTD $(4.7 \pm 0.4 \mathrm{~nm})$ and neurofilament-M CTD $(4.7 \pm 0.4 \mathrm{~nm})$, where the error arises from the range of persistence length values. Assuming each molecule occupies a sphere of radius $R_{g}$, the area it occupies on the membrane surface can be approximated as, area $=\pi R_{\mathrm{g}}^{2}$, yielding the following estimates of occupied area: AP180 CTD $\left(91 \pm 16 \mathrm{~nm}^{2}\right)$, Epsin1 CTD $\left(69 \pm 12 \mathrm{~nm}^{2}\right)$ and neurofilament-M CTD $\left(70 \pm 12 \mathrm{~nm}^{2}\right)$.

\section{Estimation of hydrodynamic radii from 3D diffusion times. Fluorescence} correlation microscopy was used to quantify the diffusivity of intrinsically disordered protein domains in dilute, 3D solution. The Stokes-Einstein theory for diffusion of spherical particles in dilute viscous solution can be used to derive first-order estimates of hydrodynamic radius from the measured values of diffusivity. The Stokes-Einstein equation states that $D=\frac{k_{\mathrm{B}} T}{6 \pi \mu R_{\mathrm{R}}}$, where $D$ represents diffusivity, $\mu$ represents the solution viscosity, $R_{\mathrm{g}}$ represents the radius of gyration of the protein molecule, $k_{\mathrm{B}}$ represents Boltzmann's constant and $T$ represents temperature. We estimated $\mu$ as approximately the viscosity of water at $17^{\circ} \mathrm{C}$ and atmospheric pressure. Rhodamine $6 \mathrm{G}$ was used as a reference molecule for solution FCS, where its diffusivity has been independently reported, $3.3 \times 10^{-6} \mathrm{~cm}^{2} \mathrm{~s}^{-1}$ (ref. 62), to relate the time of diffusion for Rhodamine $6 \mathrm{G}$ as a ratio for the time of diffusion for each of our proteins. The diffusivities were thus determined from time of diffusion measurements as follows: ENTH $\Delta \mathrm{H} 0$ $\left(7.4 \times 10^{-7} \mathrm{~cm}^{2} \mathrm{~s}^{-1}\right)$, AP180 CTD $\left(2.4 \times 10^{-7} \mathrm{~cm}^{2} \mathrm{~s}^{-1}\right)$ and Epsin $1 \mathrm{FL}$ $\left(4.4 \times 10^{-7} \mathrm{~cm}^{2} \mathrm{~s}^{-1}\right)$. With these inputs, the Stokes-Einstein equation yields the following estimates of $R_{\mathrm{g}}$ : ENTH $\Delta \mathrm{HO}(2.7 \mathrm{~nm})$, AP180 CTD $(8.4 \mathrm{~nm})$ and Epsin 1 
FL $(4.5 \mathrm{~nm})$, in reasonable agreement with estimates based on polymer theory and the crystal structure of the ENTH domain.

Calibration of the brightness of EGFP and mCherry molecules. To estimate the number of cargo molecules per clathrin coated structure, we divided the brightness of puncta in the cargo image by the measured brightness of puncta within a control sample that consisted of individual EGFP molecules adhered to a coverslip surface. To determine the brightness of individual fluorescent molecules, a dilute solution $(\sim 70 \mathrm{pM})$ of EGFP was added to an ultraclean coverslip hydrated with PBS. Images of the coverslip surface were acquired with the same laser power and camera gain settings used in all live-cell imaging experiments. A manual approach was first used, where individual fluorescent puncta corresponding to single EGFP molecules were selected from images as diffraction-limited structures of uniform brightness that were sparse on the coverslip. A Gaussian function was fit to the pixel intensity profile of lines drawn through each puncta. To determine the amplitude of the Gaussian function associated with individual molecules, the background fluorescence signal was subtracted. To validate this approach, we also used the particle detection software (described below for clathrin-coated pit detection), to measure the fluorescence amplitude of Gaussian functions fit to individual EGFP molecules. The Gaussian fluorescence amplitude from individual EGFP molecules was used to determine the number of EGFP molecules in diffraction-limited puncta corresponding to clathrin pits, simply by dividing the amplitude for the pit cargo by the amplitude of a single EGFP. This approach extrapolates from the brightness of a single EGFP molecule to determine the number of EGFP-labelled cargo molecules per pit. Since some pits contained 30 or more cargo molecules, it is important to validate this approach independently for large number of EGFP molecules. Therefore, we created SLBs with a density of EGFP molecules diffusing freely on their surfaces, in the upper concentration range of cargo molecules that are incorporated into clathrin-coated pits (at least 10 molecules per confocal focus). Scans recorded the number of photons per area per time from multiple locations over the SLB surface. These values were divided by the corresponding values for single EGFP molecules to determine the number of EGFP molecules bound to the membrane surface per area. Then, the SLB was imaged by spinning disc confocal microscopy to acquire the average fluorescence intensity. The average intensity was divided by the number of molecules per area determined by the photon scans, to provide an independent calibration of the single molecule brightness for EGFP at high density. The two methods of calibration, (i) photon counting and (ii) extrapolation from the intensity of a single EGFP molecule, agreed within $\sim 20 \%$ error when used to determine the number of EGFP molecules on the SLB surface. This test validates our approach of extrapolating from the brightness of a single EGFP molecule to determine the number of cargo molecules within clathrin-coated structures in our live-cell imaging experiments. A similar approach was followed to measure the single molecule fluorescence of mCherry to determine the number of labelled CLC molecules within clathrin structures.

Cell culture and transfection. Human RPE cells stably expressing mCherrytagged CLCs were a kind gift from Dr Allen Liu (University of Michigan) and Dr Sandra Schmid (UT Southwestern). RPE cells were grown in 1:1 F-12: DMEM media supplemented with $10 \%$ FBS, Pen/Strep/L-glutamine (100 units per ml; $100 \mu \mathrm{g} \mathrm{ml}^{-1} ; 300 \mu \mathrm{g} \mathrm{ml}^{-1}$, respectively) and incubated at $37^{\circ} \mathrm{C}$ with $5 \% \mathrm{CO}_{2}$. Cells were grown for $24 \mathrm{~h}$ on $35-\mathrm{mm}$ collagen-coated glass bottom dishes (Matek) before being transfected with $1 \mu \mathrm{g}$ chimaeric GFP cargo plasmid DNA using Fugene transfection reagent (Promega) and imaged $\sim 16 \mathrm{~h}$ after transfection. Data were collected from two dishes of cells per chimaeric cargo representing independent transfections. Spinning disc confocal images were acquired for clathrin (mCherry) and cargo (EGFP) channels at the plasma membrane surface. Cells were selected based on their level of expression, and only cells with matched levels of background expression were included in the analysis. A $20 \times 20-\mu \mathrm{m}$ crop from the image of each cell was used for analysis. Individual clathrin-coated structures were detected and analysed using particle detection software developed previously and made publically available ${ }^{47}$. Clathrin-coated structures were detected by fitting a 2D Gaussian function to the intensity profile of each putative clathrin-coated structure, where the s.d. $(\sigma)$ of the Gaussian was determined from the point spread function of the microscope system. Signals were reported as valid if the puncta was diffraction-limited and had an amplitude significantly above the local fluorescence background signal as validated previously ${ }^{47}$. Using the subpixel locations of each clathrin puncta, a 2D Gaussian function was then fit to the fluorescence signals within the corresponding regions of the EGFP (cargo) image. Puncta in the cargo image were considered valid if their locations were within 3 s.d. of the location of a corresponding structure in the clathrin signal. To ensure that only puncta with sufficient signal were analysed, we only included puncta with a cargo intensity 1 s.d. above the local background fluorescence signal.

\section{References}

1. McMahon, H. T. \& Gallop, J. L. Membrane curvature and mechanisms of dynamic cell membrane remodelling. Nature 438, 590-596 (2005).

2. Kirchhausen, T. Bending membranes. Nat. Cell Biol. 14, 906-908 (2012).
3. Jackson, L. P. et al. A large-scale conformational change couples membrane recruitment to cargo binding in the AP2 clathrin adaptor complex. Cell 141, $1220-1229$ (2010).

4. Crowther, R. A., Finch, J. T. \& Pearse, B. M. On the structure of coated vesicles. J. Mol. Biol. 103, 785-798 (1976).

5. Ungewickell, E. \& Branton, D. Assembly units of clathrin coats. Nature 289, 420-422 (1981).

6. Fotin, A. et al. Molecular model for a complete clathrin lattice from electron cryomicroscopy. Nature 432, 573-579 (2004).

7. Boettner, D. R., Chi, R. J. \& Lemmon, S. K. Lessons from yeast for clathrin-mediated endocytosis. Nat. Cell Biol. 14, 2-10 (2012).

8. Ford, M. G. et al. Curvature of clathrin-coated pits driven by epsin. Nature 419, 361-366 (2002).

9. Frost, A., Unger, V. M. \& De Camilli, P. The BAR domain superfamily: membrane-molding macromolecules. Cell 137, 191-196 (2009).

10. Lafer, E. M. Clathrin-protein interactions. Traffic 3, 513-520 (2002).

11. Kalthoff, C., Alves, J., Urbanke, C., Knorr, R. \& Ungewickell, E. J. Unusual structural organization of the endocytic proteins AP180 and epsin 1. J. Biol. Chem. 277, 8209-8216 (2002).

12. Zhuo, Y. et al. Dynamic interactions between clathrin and locally structured elements in a disordered protein mediate clathrin lattice assembly. J. Mol. Biol. 404, 274-290 (2010).

13. Scheele, U. et al. Molecular and functional characterization of clathrin- and AP-2-binding determinants within a disordered domain of auxilin. J. Biol. Chem. 278, 25357-25368 (2003).

14. Dafforn, T. R. \& Smith, C. J. Natively unfolded domains in endocytosis: hooks, lines and linkers. EMBO Rep. 5, 1046-1052 (2004).

15. Pietrosemoli, N., Pancsa, R. \& Tompa, P. Structural disorder provides increased adaptability for vesicle trafficking pathways. PLoS Comput. Biol. 9, e1003144 (2013).

16. Stachowiak, J. C., Hayden, C. C. \& Sasaki, D. Y. Steric confinement of proteins on lipid membranes can drive curvature and tubulation. Proc. Natl Acad. Sci. USA 107, 7781-7786 (2010).

17. Stachowiak, J. C. et al. Membrane bending by protein-protein crowding. Nat. Cell Biol. 14, 944-949 (2012)

18. Carnahan, N. F. \& Starling, K. E. Equation of State for Nonattracting Rigid Spheres. J. Chem. Phys. 51, 635-63 (1969).

19. Carignano, M. A. \& Szleifer, I. On the Structure and Pressure of Tethered Polymer Layers in Good Solvent. Macromolecules 28, 3197-3204 (1995).

20. Scheve, C. S., Gonzales, P. A., Momin, N. \& Stachowiak, J. C. Steric pressure between membrane-bound proteins opposes lipid phase separation. J. Am. Chem. Soc. 135, 1185-1188 (2013).

21. Montesano, G., Bartucci, R., Belsito, S., Marsh, D. \& Sportelli, L. Lipid membrane expansion and micelle formation by polymer-grafted lipids: scaling with polymer length studied by spin-label electron spin resonance. Biophys. J. 80, 1372-1383 (2001).

22. Copic, A., Latham, C. F., Horlbeck, M. A., D’Arcangelo, J. G. \& Miller, E. A. ER cargo properties specify a requirement for COPII coat rigidity mediated by Sec13p. Science 335, 1359-1362 (2012).

23. Wu, T., Shi, Z. \& Baumgart, T. Mutations in BIN1 associated with centronuclear myopathy disrupt membrane remodeling by affecting protein density and oligomerization. PLOS ONE 9, e93060 (2014).

24. Goh, S. L., Wang, Q., Byrnes, L. J. \& Sondermann, H. Versatile membrane deformation potential of activated pacsin. PLOS ONE 7, e51628 (2012).

25. Vanlandingham, P. A. et al. AP180 couples protein retrieval to clathrin mediated endocytosis of synaptic vesicles. Traffic 15, 433-450 (2014).

26. Jiang, Z., de Messieres, M. \& Lee, J. C. Membrane remodeling by alpha-synuclein and effects on amyloid formation. J. Am. Chem. Soc. 135, 15970-15973 (2013)

27. Kaufmann, A., Beier, V., Franquelim, H. G. \& Wollert, T. Molecular mechanism of autophagic membrane-scaffold assembly and disassembly. Cell 156, 469-481 (2014)

28. Hofmann, H. et al. Polymer scaling laws of unfolded and intrinsically disordered proteins quantified with single-molecule spectroscopy. Proc. Natl Acad. Sci. USA 109, 16155-16160 (2012).

29. Boucrot, E. et al. Membrane fission is promoted by insertion of amphipathic helices and is restricted by crescent BAR domains. Cell 149, 124-136 (2012).

30. Phillips, R., Kondev, J. \& Theriot, J. Physical Biology of the Cell (Garland Science, 2009).

31. Muramatsu, N. \& Minton, A. P. Tracer diffusion of globular proteins in concentrated protein solutions. Proc. Natl Acad. Sci. USA 85, 2984-2988 (1988).

32. Paulick, M. G., Wise, A. R., Forstner, M. B., Groves, J. T. \& Bertozzi, C. R. Synthetic analogues of glycosylphosphatidylinositol-anchored proteins and their behaviour in supported lipid bilayers. J. Am. Chem. Soc. 129, 11543-11550 (2007).

33. Knight, J. D., Lerner, M. G., Marcano-Velazquez, J. G., Pastor, R. W. \& Falke, J. J. Single molecule diffusion of membrane-bound proteins: window into lipid contacts and bilayer dynamics. Biophys. J. 99, 2879-2887 (2010).

34. Ackerson, B. J. \& Fleishman, L. Correlations for dilute hard-core suspensions. J. Chem. Phys. 76, 2675-2679 (1982). 
35. Blondeau, F. et al. Tandem MS analysis of brain clathrin-coated vesicles reveals their critical involvement in synaptic vesicle recycling. Proc. Natl Acad. Sci. USA 101, 3833-3838 (2004).

36. Prasad, K. \& Lippoldt, R. E. Molecular characterization of the AP180 coated vesicle assembly protein. Biochemistry 27, 6098-6104 (1988).

37. Cheng, Y., Boll, W., Kirchhausen, T., Harrison, S. C. \& Walz, T. Cryo-electron tomography of clathrin-coated vesicles: structural implications for coat assembly. J. Mol. Biol. 365, 892-899 (2007).

38. Morgan, J. R. et al. A role for the clathrin assembly domain of AP180 in synaptic vesicle endocytosis. J. Neurosci. 19, 10201-10212 (1999).

39. Zhang, B. et al. Synaptic vesicle size and number are regulated by a clathrin adaptor protein required for endocytosis. Neuron 21, 1465-1475 (1998).

40. Nonet, M. L. et al. UNC-11, a Caenorhabditis elegans AP180 homologue, regulates the size and protein composition of synaptic vesicles. Mol. Biol. Cell 10, 2343-2360 (1999).

41. Petralia, R. S. et al. Reduction of AP180 and CALM produces defects in synaptic vesicle size and density. Neuromol. Med. 15, 49-60 (2013).

42. Schmid, E. M. \& McMahon, H. T. Integrating molecular and network biology to decode endocytosis. Nature 448, 883-888 (2007).

43. Geisler, N., Kaufmann, E., Fischer, S., Plessmann, U. \& Weber, K. Neurofilament architecture combines structural principles of intermediate filaments with carboxy-terminal extensions increasing in size between triplet proteins. EMBO J. 2, 1295-1302 (1983).

44. Brown, H. G. \& Hoh, J. H. Entropic exclusion by neurofilament sidearms a mechanism for maintaining interfilament spacing. Biochemistry 36, 15035-15040 (1997).

45. Geisler, N. \& Weber, K. Self-assembly in Vitro of the 68,000 molecular weight component of the mammalian neurofilament triplet proteins into intermediate-sized filaments. J. Mol. Biol. 151, 565-571 (1981).

46. Kumar, S., Yin, X. H., Trapp, B. D., Hoh, J. H. \& Paulaitis, M. E. Relating interactions between neurofilaments to the structure of axonal neurofilament distributions through polymer brush models. Biophys. J. 82, 2360-2372 (2002).

47. Aguet, F., Antonescu, C. N., Mettlen, M., Schmid, S. L. \& Danuser, G. Advances in analysis of low signal-to-noise images link dynamin and AP2 to the functions of an endocytic checkpoint. Dev. Cell 26, 279-291 (2013).

48. Boll, W. et al. Sequence requirements for the recognition of tyrosine-based endocytic signals by clathrin AP-2 complexes. EMBO J. 15, 5789-5795 (1996).

49. Weigel, A. V., Tamkun, M. M. \& Krapf, D. Quantifying the dynamic interactions between a clathrin-coated pit and cargo molecules. Proc. Natl Acad. Sci. USA 110, E4591-E4600 (2013).

50. Schmid, E. M. Role of the AP2 beta-appendage hub in recruiting partners for clathrin-coated vesicle assembly. Plos Biol. 4, e262 (2006).

51. Lim, R. Y. et al. Flexible phenylalanine-glycine nucleoporins as entropic barriers to nucleocytoplasmic transport. Proc. Natl Acad. Sci. USA 103, 9512-9517 (2006).

52. Patel, S. S., Belmont, B. J., Sante, J. M. \& Rexach, M. F. Natively unfolded nucleoporins gate protein diffusion across the nuclear pore complex. Cell 129, 83-96 (2007).

53. Curry, F. E. \& Adamson, R. H. Endothelial glycocalyx: permeability barrier and mechanosensor. Ann. Biomed. Eng. 40, 828-839 (2012).

54. Uchida, K., Otsuka, H., Kaneko, M., Kataoka, K. \& Nagasaki, Y. A reactive poly(ethylene glycol) layer to achieve specific surface plasmon resonance sensing with a high $\mathrm{S} / \mathrm{N}$ ratio: The substantial role of a short underbrushed PEG layer in minimizing nonspecific adsorption. Anal. Chem. 77, 1075-1080 (2005).

55. Collins, B. M., McCoy, A. J., Kent, H. M., Evans, P. R. \& Owen, D. J. Molecular architecture and functional model of the endocytic AP2 complex. Cell 109, 523-535 (2002)
56. Mettlen, M., Loerke, D., Yarar, D., Danuser, G. \& Schmid, S. L. Cargo- and adaptor-specific mechanisms regulate clathrin-mediated endocytosis. J. Cell Biol. 188, 919-933 (2010).

57. Liu, A. P., Aguet, F., Danuser, G. \& Schmid, S. L. Local clustering of transferrin receptors promotes clathrin-coated pit initiation. J. Cell Biol. 191, 1381-1393 (2010).

58. Dannhauser, P. N. \& Ungewickell, E. J. Reconstitution of clathrin-coated bud and vesicle formation with minimal components. Nat. Cell Biol. 14, 634-639 (2012).

59. Yan, Y., Jensen, K. \& Brown, A. The polypeptide composition of moving and stationary neurofilaments in cultured sympathetic neurons. Cell Motil. Cytoskeleton 64, 299-309 (2007).

60. Angelova, M. \& Dimitrov, D. Liposome electroformation. Faraday Discuss. Chem. Soc. 81, 303-311 (1986).

61. Zhou, H. X. Polymer models of protein stability, folding, and interactions. Biochemistry 43, 2141-2154 (2004).

62. Muller, C. B. et al. Precise measurement of diffusion by multi-color dual-focus fluorescence correlation spectroscopy. Europhys. Lett. 83, 1-5 (2008).

\section{Acknowledgements}

J.C.S. acknowledges support from the National Institutes of Health (1R01GM112065 to Stachowiak in support of Busch, Houser, and Hayden) as well as startup funding from The University of Texas at Austin. E.M.L. acknowledges support from the National Institutes of Health (NIH-NS029051 to Lafer). We thank Dr Ernst Ungewickell (Hannover Medical School) for providing the Epsinl CTD and AP180 CTD plasmids, Dr Thomas Kirchhausen (Harvard Medical School) for providing a transferrin receptor plasmid and Dr Anthony Brown (Ohio State University) for making the neurofilamentM plasmid available through Addgene. We thank Dr Dwight Romanovicz and the ICMB Microscopy Facility at UT Austin for assistance with electron microscopy. We thank Dr Allen Liu (University of Michigan) and Dr Sandra Schmid (UT Southwestern Medical School) for providing the RPE cell line stably expressing mCherry-labelled CLC used in this study, as well as for helpful advice using the clathrin pit detection software, freely provided by the lab of Dr Gaudenz Danuser (Harvard Medical School). We thank Dr Terry O'Halloran (UT Austin) for feedback on this project, as well as undergraduate researchers Jerin Jose, Saad Jafri and Brian Li for assistance with preliminary experiments.

\section{Author contributions}

D.J.B., J.R.H., C.C.H. and J.C.S. performed experiments and performed data analysis. All authors designed experiments, discussed the results and conclusions, and wrote the manuscript.

\section{Additional information}

Supplementary Information accompanies this paper at http://www.nature.com/ naturecommunication

Competing financial interests: The authors declare no competing financial interests

Reprints and permission information is available online at http://npg.nature.com/ reprintsandpermissions/

How to cite this article: Busch, D. J. et al. Intrinsically disordered proteins drive membrane curvature. Nat. Commun. 6:7875 doi: 10.1038/ncomms8875 (2015)

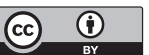

This work is licensed under a Creative Commons Attribution 4.0 International License. The images or other third party material in this article are included in the article's Creative Commons license, unless indicated otherwise in the credit line; if the material is not included under the Creative Commons license, users will need to obtain permission from the license holder to reproduce the material To view a copy of this license, visit http://creativecommons.org/licenses/by/4.0/ 